\title{
PO054
}

\section{DEVELOPMENT OF WHITENESS INDEX FOR FACIAL COLOUR}

\author{
Mengmeng Wang et al.
}

DOI 10.25039/x46.2019.PO054

from

CIE x046:2019

\author{
Proceedings \\ of the \\ 29th CIE SESSION \\ Washington D.C., USA, June 14 - 22, 2019 \\ (DOI 10.25039/x46.2019)
}

The paper has been presented at the 29th CIE Session, Washington D.C., USA, June 14-22, 2019. It has not been peer-reviewed by CIE.

\section{(C) CIE 2019}

All rights reserved. Unless otherwise specified, no part of this publication may be reproduced or utilized in any form or by any means, electronic or mechanical, including photocopying and microfilm, without permission in writing from CIE Central Bureau at the address below. Any mention of organizations or products does not imply endorsement by the CIE.

This paper is made available open access for individual use. However, in all other cases all rights are reserved unless explicit permission is sought from and given by the CIE.

CIE Central Bureau

Babenbergerstrasse 9

A-1010 Vienna

Austria

Tel.: +4317143187

e-mail: ciecb@cie.co.at

www.cie.co.at 


\title{
DEVELOPMENT OF WHITENESS INDEX FOR FACIAL COLOUR
}

\author{
Mengmeng Wang ${ }^{1,2}$, Kaida $\mathrm{Xiao}^{2}$, Michael Pointer ${ }^{2}$ \\ 1 Jiangnan University, Wuxi, P.R.CHINA \\ 2 University of Leeds, Leeds. UNITED KINGDOM
}

k.xiao1@leeds.ac.uk

DOI 10.25039/x46.2019.PO054

\begin{abstract}
Skin whiteness is an attribute that is frequently used to describe skin colour. This study investigated the relationship between perceived skin whiteness and associated physical measurements. A psychophysical experiment was conducted with 40 participants (20 Chinese and 20 Caucasian). Images of eighty real human faces, from the Leeds-Liverpool skin colour database (LLSD), were used in the experiment. The correlation between the experimental results and the CIELAB colorimetric scales showed that skin whiteness has a relatively strong correlation with the CIELAB $L^{*}$ and $b^{*}$ values, and less correlation with CIELAB $a^{*}$. Two whiteness related formulas, ITA ${ }^{\circ}$ and Depth $\left(D^{*}{ }_{a b}\right)$ were tested in this study. The ITA ${ }^{\circ}$ formula gave a better estimation of the perceived skin whiteness than the Depth formula, but gave poor prediction for dark skin colours. A skin whiteness index was developed, which gave good estimation of our perceived skin whiteness data.
\end{abstract}

Keywords: Skin colour, facial whiteness, skin colour data

\section{Introduction}

Skin colour is an obvious characteristic of human appearance and has been shown to be closely related to facial attractiveness (Wang, et.al, 2017). Skin whiteness is frequently used to describe the skin colour in the cosmetics industry. It is also considered as an important element of Asian beauty, and skincare and cosmetic products have been developed to help consumers to increase the whiteness of their skin. Skin whiteness also influences the digital imaging industry, which has led to the development of image processing algorithms to output preferred white facial skin colour images to meet the needs of the Asian market.

Whiteness is an important colour attribute in many other industries, such as in paper manufacture and the paint quality control, and also in current colour research. Many indices have been developed to evaluate perceived whiteness. For example, the CIE whiteness index (WIC) is a commonly used index that is often cited in standards (e.g. ISO, 2004). However, like many other whiteness indices in common use, it can only be used within a defined range of chroma and hue (or tint). Apart from these whiteness indices, whiteness scales for describing colours within a larger hue and chroma range have been developed. For example, the Berns' Depth scale extends the utility of CIELAB in terms of the "paleness" and "depth" of the colour (Berns, 2014). This scale can be used to describe the relationship between the colour and the neutral (white) colour, which is similar to the concept of whiteness. The formula of the Depth is given in Equation 1

$$
D_{a b}^{*}=\sqrt{\left(100-L^{*}\right)^{2}+\left(C_{a b}^{*}\right)^{2}} \quad \text { Equation } 1
$$

Thus, the Depth is calculated as the distance between the whitest colour $\left(L^{*}=100, C^{*}{ }_{a b}=0\right)$ and the sample colour in $L^{*}, C^{*}$ ab space. It has been shown to give good predictions of the colourant strength of a colour. Similar to the other whiteness scales, such as Adam's whiteness scale (Adams, 2010), Cho's whiteness scale (Cho, et al., 2017), this scale was developed based on paper colour samples that included a limited set of skin colour patches. The Individual Typology Angle $\left(\mathrm{ITA}^{\circ}\right.$ ) was developed to determine the melanisation of the skin (Chardon, et al., 1991) and it is commonly used to determine the Fitzpatrick skin prototype types of the skin for sun tanning. The formula, which is based on the CIELAB $L^{*}$ and $b^{*}$ values is shown in Equation 2. 


$$
I T A^{\circ}=\operatorname{ATAN} \frac{\left(L^{*}-50\right)}{b^{*}} \times \frac{180}{3.14159} \quad \text { Equation } 2
$$

A higher $\mathrm{ITA}^{\circ}$ value indicates a lighter skin colour. This formula was developed based on the measurements of a set of Caucasian skin colours and visual assessment was not included.

The real human skin colour is unevenly distributed on a usually curved surface and often with an inherent texture. Even though there have been many studies to investigate the perceived whiteness of the skin, in many instances the results have been inconclusive, and this has made comparison between these results difficult. The aim of this study is to investigate the relationship between the perceived whiteness and the measurement of skin colour. Two whiteness scales from previous research have been evaluated and, in addition, a new skin whiteness formula is proposed.

\section{Experimental Design}

A psychophysical experiment was carried out to investigate the relationship between the perceived skin whiteness and measurements of skin colour. The measurement data in this study were obtained from the Liverpool-Leeds skin-colour database (LLSD), which includes facial images and associated skin colour reflectance data for 188 subjects (including four different ethnicities). In the database, for each subject, ten locations (facial and body locations) were identified and their spectral reflectance were measured using a telespectroradiometer (TSR) with the human subject placed in a Verivide viewing cabinet to provide controlled illumination (Wang, et al. 2018). Facial images of each subject were captured using a Nikon digital SLR camera. A reflectance estimation algorithm (Xiao, et al., 2016) was used to transform the digital RGB image values to the corresponding spectral reflectance for the skin colours in each facial image. Thus, there were two sets of spectral reflectance data: measured and calculated.

In this study, eighty facial images, including 40 Caucasian females and 40 Chinese females, were randomly selected from the database. The facial area of each image was extracted to use in this study. Two facial images, including a target face and a reference face, were displayed side by side on a calibrated display. Figure 1 shows the observer interface for the psychophysical experiment. The reference face was an average face derived from the 40 facial images, including 20 Caucasian and 20 Chinese females. The location (left or right) of the reference face and the target face were set to be random.

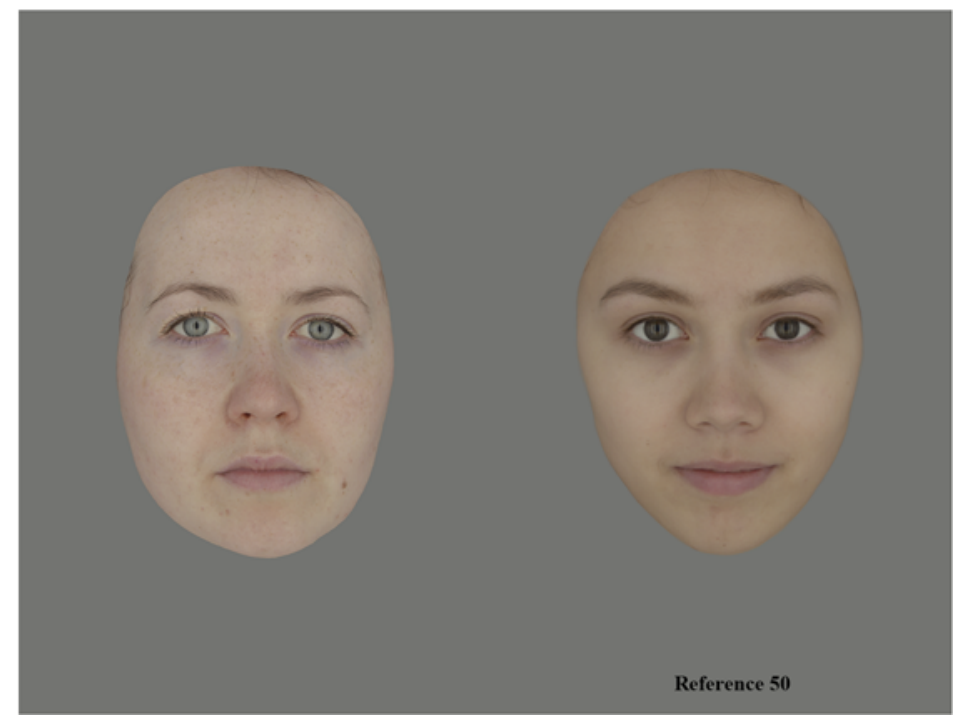

Figure 1 - The interface of the psychophysical experiment.

Forty participants, including 20 Caucasian (10 female and 10 male) and 20 Chinese (10 female and 10 male), participated in the magnitude estimation experiment. Each participant was asked to sit in front of the display, which was located in a darkened room, and estimate the whiteness of the target face in comparison with the reference face. The observers were told that the whiteness of the reference face was set to have a value of 50 . The allowed range of the 
whiteness score for the target face was set to be between 0 and 100 , where 100 represented the whitest possible skin colour according to the observer's experience. The results for the 40 observers were averaged and analysed.

\section{Results and Analysis}

\subsection{Observer inter-variation}

The root-mean-square deviation (RMSD) of the experimental results was used to determine the variation amongst the observers: the RMSD of the Caucasian and the Chinese observers were calculated separately and Figure 2 shows a bar chart of the results.

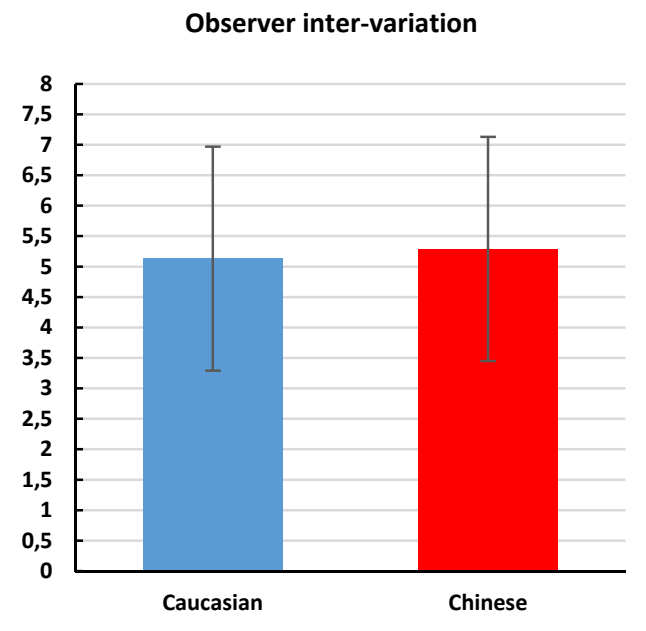

Figure 2 - The inter-variation of Caucasian (blue) and Chinese (red) observer respectively.

From this figure it can be seen that the variation amongst the Caucasian and Chinese observers was 5.13 and 5.29 , with standard deviation of 1.84 and 1.99 , respectfully; i.e. the results for the two sets of observers were very similar.

\subsection{Facial whiteness}

For each facial image, an average value of the perceived facial whiteness was obtained from the psychophysical experiment. The skin colour measurements, on the other hand, were obtained from the database via image processing. For each facial image, the colour of the skin areas, in terms of RGB values, was averaged and then transformed to spectral reflectance (Xiao et al., 2016). From these data the CIELAB colorimetric coordinates could be calculated using CIE D65 illumination and the CIE 2 degree standard observer.

Figure 3 shows charts of the perceived skin whiteness plotted as a function of the CIELAB $L^{*}$, $a^{*}$ and $b^{*}$ values of the 80 images, respectively. The correlation coefficient $(r)$ was used as a measure of the correlation between the perceived whiteness and the respective CIELAB scales.

Figure 3 shows that the psychophysical experimental results have a stronger correlation with the $L^{*}$ and $b^{*}$ values than with the $a^{*}$ value. The $r$ values are $0.90,0.31$ and 0.90 , for $L^{*}, a^{*}$ and $b^{*}$ respectively. This indicates that the perceived skin whiteness has a stronger correlation with the perceived yellowness than with perceived redness. 
$\mathrm{L}^{*}$

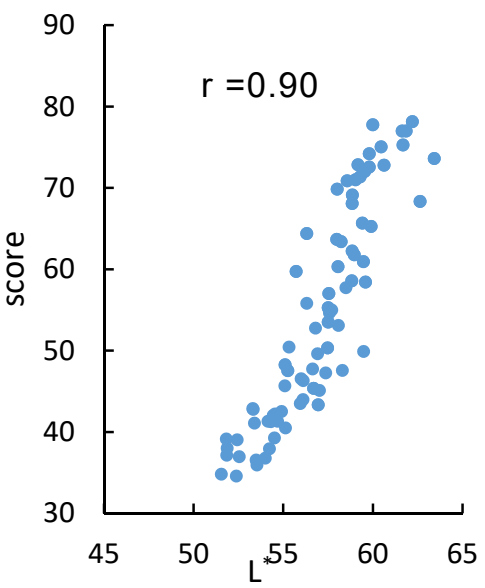

$a^{*}$

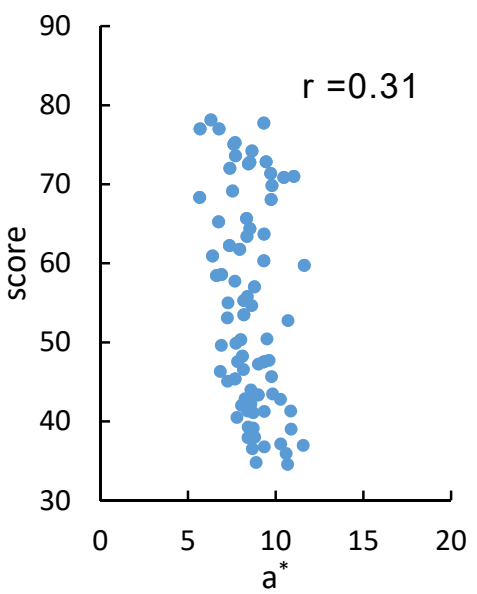

$b^{*}$

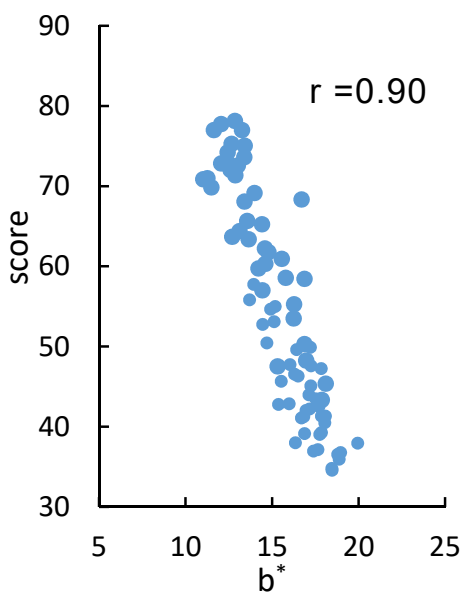

Figure 3 - The relationship between the experimental results and CIELAB.

Two whiteness related scales, ITA ${ }^{\circ}$ and the Bern's Depth scale, were evaluated in this study. The CIELAB colorimetric values were calculated from the image based measurements and used to calculate the $\mathrm{ITA}^{\circ}$ and Depth of all 80 facial images. R-squared was used to evaluate the performance of these scales for skin whiteness prediction. Figure 4 shows charts of the experimental results for the two scales, the ITA ${ }^{\circ}$ and Depth, respectively.

$\mathrm{ITA}^{\circ}$

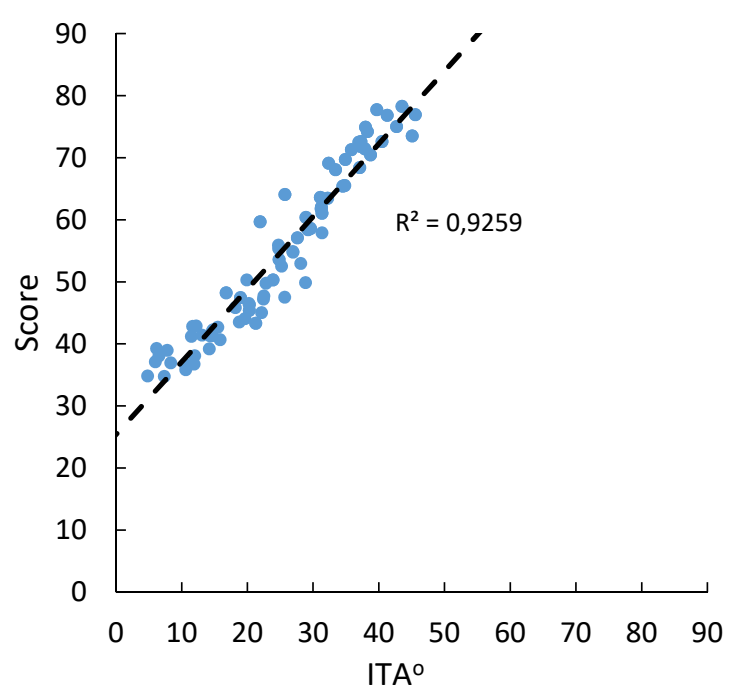

Depth

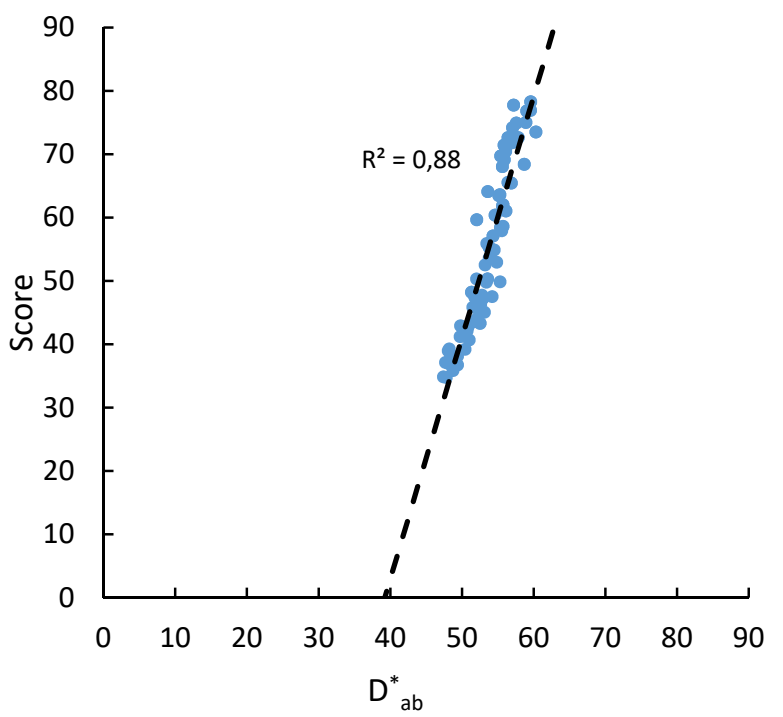

Figure 4 - The observer's whiteness response as a function of (left) the ITA ${ }^{\circ}$ scale, and (right) the Bern's Depth scale.

Figure 4 shows that both scales give reasonable correlation with the experimental results: the distribution of the data points in Figure 4(a) however, appears more scattered than that in Figure $4(b)$. This is reflected in the associated R-squared values of 0.93 and 0.88 , respectively, i.e. the ITA ${ }^{\circ}$ scale gave better prediction than the Depth scale but it only within a certain ITA ${ }^{\circ}$ range. A skin whiteness value below 40 in the experiments gave a similar ITA ${ }^{\circ}$ value, approximately 10. This can be attributed to the fact that the $\mathrm{ITA}^{\circ}$ scale uses an angular measure to represent the skin whiteness. Also, the ITA ${ }^{\circ}$ scale only includes the CIELAB $L^{*}$ and $b^{*}$ values for whiteness prediction. Some previous research has found that the redness of the skin correlates with the 
perceived skin whiteness (Yano and Hashimoto, 1997). Thus, including a contribution from the CIELAB $a^{*}$ value on the formula for ITA $^{\circ}$ might improve the correlation even further.

For the Depth scale, the absolute distance between the whitest colour and sample colour is used to determine the degree of depth or paleness: a smaller Depth value implies a whiter colour. Figure $5(\mathrm{a})$ illustrates the Depth $\left(D^{*} \mathrm{ab}\right)$ by the red arrow with the Chinese and Caucasian skin colours in the LLSD. From this figure it can be seen that the distribution of the skin whiteness (Chinese and Caucasian) is similar to the Depth scale, where the skin colour with higher $\mathrm{L}^{*}$ and lower $\mathrm{C}^{*}{ }_{\mathrm{ab}}$ appears to be whiter.

Therefore, a skin whiteness index has been developed based on the Depth scale. The Depth formula is constructed to determine the depth/paleness for all colours and uses an $L^{*}$ value equal to 100 to represent the whitest colour. For skin colours, the whitest colour can be determined from the LLSD skin colour database. Based on the facial skin colour of Caucasian and Chinese images in the database, as shown in Figure 5, the whitest skin colour can be determined by the trendline of the skin colour cluster. The intersection of this trend line with the $L^{*}$ axis can be considered as the whitest skin colour of the Chinese and Caucasian group. Thus, the whitest skin colour has an $L^{*}$ value of 80.7 . The formula for a skin whiteness index is given in Equation 3.

$$
W_{\text {skin }}=\sqrt{\left(80.7-L^{*}\right)^{2}+\left(C_{a b}^{*}\right)^{2}} \quad \text { Equation 3 }
$$

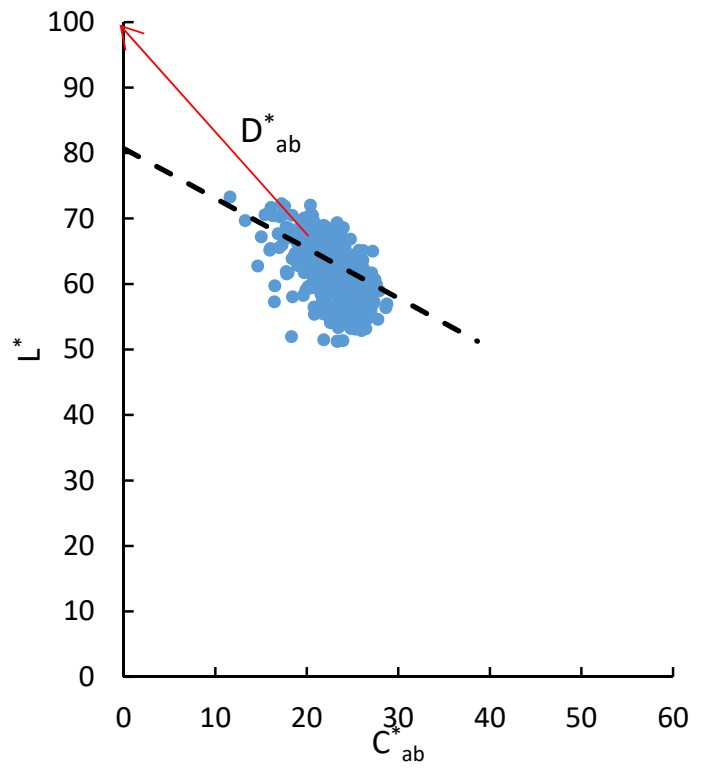

Figure 5 - The distribution of the skin colour of the Caucasian and Chinese subjects in CIELAB $L^{*}, C^{*}$ space. 
new skin whiteness scale

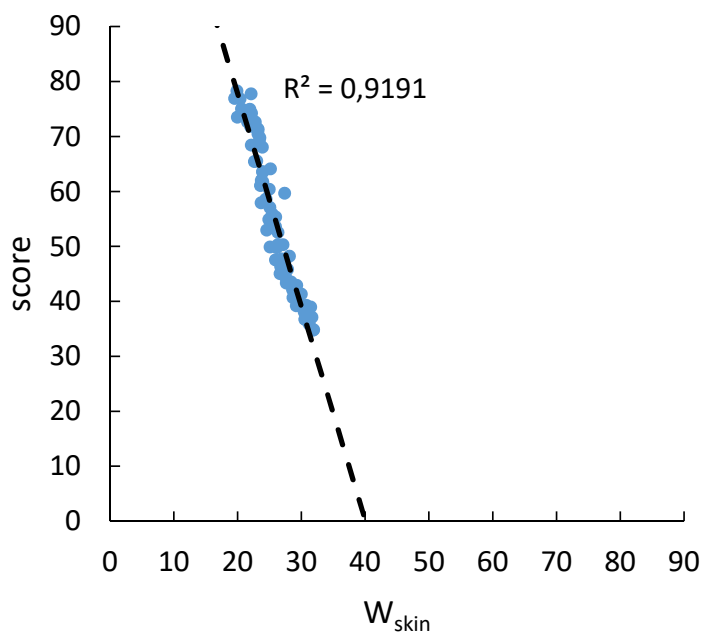

Figure 6 - The observer's whiteness response as a function of the new skin whiteness scale.

Figure 6 shows the psychophysical experimental data plotted as a function of the new skin whiteness index. The R-square of the experimental results with the new whiteness formula for skin is 0.92 , which shows that this skin whiteness index gave a good performance of estimation.

\section{Conclusion}

This study aimed to investigate the relationship between perceived skin whiteness and the physical colour of the skin. A psychophysical experiment was carried out to determine this relationship. The experimental results showed that the perceived skin whiteness has a strong correlation with the lightness and the yellowness of the skin. Two whiteness related scales, $\mathrm{ITA}^{\circ}$ and Depth were examined. From the R-squared value it was shown that the $\mathrm{ITA}^{\circ}$ scale had better performance than the Depth scale. But the ITA $^{\circ}$ scale showed poor linearity for darker skin colours. A new whiteness scale for skin colour was developed based on an extension of the Bern's Depth scale. This new scale shows gave improved performance with Rsquared values improved from 0.88 to 0.92 , which is very close to the performance of the ITA ${ }^{\circ}$ scale.

\section{References}

Yano, T. and Hashimoto, K. (1997). Preference for Japanese complexion color under illumination. Color Res. Appl. 22(4), pp.269-274.

Adams, L. W. (2010). Whiteness, chromaticness, and blackness symmetries for CIELAB, with a numerical example. Color Res. Appl., 35(4), 319-323.

Berns, R.S. (2014). Extending CIELAB: Vividness, $\mathrm{V}_{a b}{ }^{*}$, depth, $\mathrm{D}_{\mathrm{ab}}{ }^{*}$, and clarity, $\mathrm{T}_{\mathrm{ab}}$. Color Res. Appl. 39(4), pp.322-330.

Chardon A., Cretois I., and Hourseau C. (1991). Skin colour typology and suntanning pathways. Int. J. Cosmet. Sci. 13, pp.191-208.

Cho YJ, Ou LC, Cui G, Luo R. (2017). New colour appearance scales for describing saturation, vividness, blackness, and whiteness. Color Res. Appl. 42(5), pp.552-63.

Wang M, Xiao K, Luo MR, Pointer M, Cheung V, Wuerger S. (2018). An investigation into the variability of skin colour measurements. Color Res. Appl., 43(4), pp. 458-470 
Xiao K, Zhu Y, Li C, Connah D, Yates JM, Wuerger S. (2016). Improved method for skin reflectance reconstruction from camera images. Optics Express. 24(13), pp. 14934-14950

ISO (2004), ISO 11475:2004 Paper and board - determination of CIE whiteness, D65/10o (outdoor daylight). International Organisation for Standardisation, 2004. p. 12.

Wang, M., Luo, MR., Xiao, K. (2017) The impact of skin colour on facial impressions. AIC Conference 2017. 